\title{
Finite Element Simulation of Shot Peening - A Method to Evaluate the Influence of Peening Parameters on Surface Characteristics
}

\author{
Jochen Schwarzer, Volker Schulze, Otmar Vöhringer \\ Institut für Werkstoffkunde I, University of Karlsruhe (TH), Karlsruhe, Germany
}

\section{$1 \quad$ Introduction}

Shot peening, known for its potential to improve fatigue strength of metallic parts, can be seen as a multiple and progressively repeated elastic-plastic interaction between the surface and the shots. With each impact the target undergoes local plastic deformation while the shot is moving into the material. After the contact between the target and the shot has ceased, compressive residual stresses remain at the surface and small tensile residual stresses in the inside.

Developing a model to analyze the process of shot peening is useful for several reasons; to be able to predict the material state after peening without having to conduct costly experiments and to be able to optimize peening processes. In contrast to prior studies found in the literature [1-4], a more detailed approch by simulating several single impacts on a 3-dimensional surface is chosen to model the shot peening process.

\section{$2 \quad$ Finite Element Modelling}

\subsection{Model Geometry and Boundary Conditions}

The model used for the shot-peening analyses was realized in ABAQUS/Explicit and consists of an infinite steel sheet of thickness $0.85 \mathrm{~mm}$ and multiple half-spheres. The target is represented by a three-dimensional mesh of $1.5 \times 1.5 \times 0.85 \mathrm{~mm}$, surrounded by infinite elements. The use of infinite elements provides "quiet" boundaries by minimizing the reflection of dilatational and shear wave energy back into the finite element mesh. The boundary conditions on the target's base fix the model in z-direction. The mesh consists of 372000 8-node linear brick elements with reduced integration and hourglass control. In order to achieve sufficient discretisation it is graded in all three directions so that the smallest element occurs in the middle of the target area with an element size of $0.008 \mathrm{~mm}$.

Based on measurements of the cast steel shot used for experimental verification, half spherical rigid surfaces of diameter $0.56 \mathrm{~mm}$ are used to model the shot. Each rigid surface is connected to a point mass and a rotary inertia element providing the properties of a full sphere. Figure 1 shows the mesh and one half-sphere after the impact of 19 shots. 


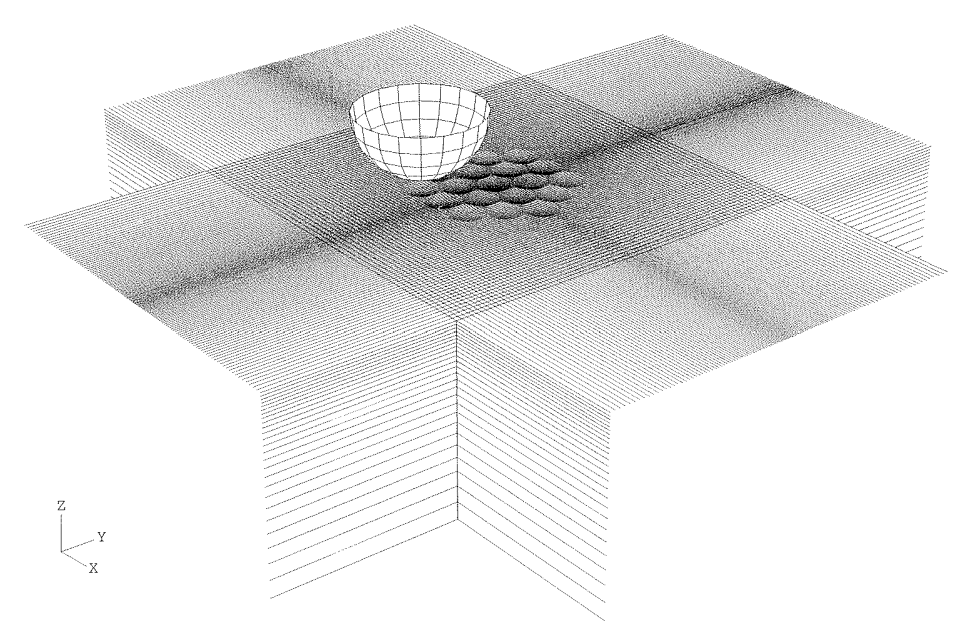

Figure 1: Discretised model used in shot peening simulation

\subsection{Material Properties}

Great importance was attached to the description of the target material properties. The quenched and tempered steel AISI 4140 (German grade: $42 \mathrm{CrMo} 4, R_{e}=1263 \mathrm{MPa}, R_{m}=1373 \mathrm{MPa}$ ) was chosen therefore. A constitutive law which describes the influence of temperature and strain-rate on the flow stress on the basis of thermally activated dislocation slip was implemented into the finite element code using a user subroutine VUMAT. Accordingly, the flow stress $\sigma_{0}$, depending on strain-rate $\bar{\varepsilon}_{0}$ and temperature T, is calculated using [5]:

$$
\sigma_{0}=\sigma_{G}+\sigma_{0}^{*}\left[1-\left(\frac{k T \ln \left(\bar{\varepsilon}_{0} / \bar{\varepsilon}\right)}{\Delta G_{0}}\right)^{n}\right]^{m}
$$

where $\sigma_{G}$ is the athermal proportion of the flow stress, $\Delta G_{0}$ is an activation enthalpy and $\sigma_{0}{ }^{*}, \bar{\varepsilon}_{0}, \mathrm{n}, \mathrm{m}$ are further material dependent parameters describing the thermal flow stress component. $\mathrm{k}$ is the Boltzmann constant. The work hardening behavior was modelled using a "generalized voce" constitutive equation according to [6]:

$$
\sigma_{G}=\sigma_{G 0}+\left(\sigma_{l}+\theta_{l} \varepsilon\right)\left[1-\exp \left(-\theta_{0} \varepsilon / \sigma_{l}\right)\right]
$$

where $\sigma_{G 0}$ and $\theta_{0}$ describe the initial yield stress and hardening rate, while $\sigma_{l}$ and $\theta_{l}$ determine the asymptotic characteristics of the hardening. The material constants were determined by a numerical fit of data obtained from tensile tests at different temperatures and strain-rates to the material law similarly to [5]. By comparing the shape of simulated and experimentally produced 
shot impacts, the material law could be validated. To describe the contact between shot and target, isotropic Coulomb friction with a coefficient of friction $\mu=0.4$ is used.

The cast steel shot is modeled as a rigid body with a mass density of $7.85 \mathrm{~g} / \mathrm{cm}^{3}$. Elastic or plastic behavior of the shot is not beeing considered in the modelling.

\subsection{Impact Order of the Shot}

To achieve a realistic modelling of a shot peening process with full coverage an arrangement of the spheres was chosen that provides a closest packed dimple pattern on the surface (Fig. 2). The gray marked inner area which can be approximated with a circle was used for the calculation of residual stress profiles.

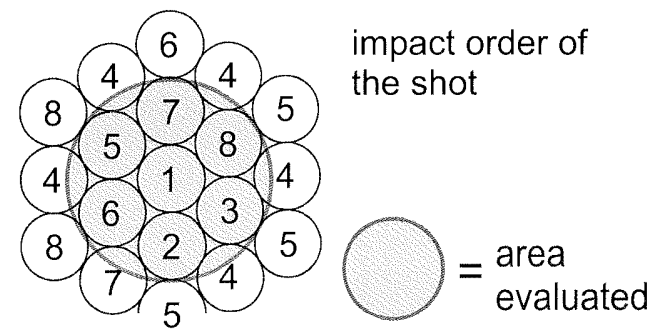

Figure 2: Arrangement and order of impacts as well as area evaluated to calculate the residual stress profile

As will be shown later, the fact that the shots impact one after the other instead of impacting simultaneously has great influence on the developing residual stresses. Simplifying a model by using its symmetry and modelling only a part of it does always imply several shots impacting simultaneously. To prevent this, symmetry wasn't considered in the modelling. The impacts in the inner circle occur one after the other. For computational costs it was allowed that non adjacent shots in the outer circle impact simultaneously.

An other aspect concerning the impact order is the number of predecessors and successors around each dimple. In the chosen arrangement each dimple is surrounded by 6 further dimples. Each of the seven inner dimples, which were used for the calculation of residual stress profiles, has a different number of predecessors and successors.

\subsection{Analysis of Residual Stress Profiles}

In the scale of the dimple size, shot peened surfaces don't show a uniform distribution of residual stresses. This is shown by experimental results [7] and will later be shown in the performed analyses of seven impacting spheres.

Residual stress profiles, usually measured by X-ray diffraction, give an average of stresses in an area covered by the X-ray. To achieve comparable analysis results a mean of residual stresses in a representative area has to be calculated at each depth. The chosen area in the finite element model consists of a circle enclosing the seven inner dimples in figure 2. Within this area an aver- 
age of the residual stresses parallel to the surface weightened with the element-size is calculated. To exclude a direction dependence resulting from non-perpendicular shots, the mean of residual stresses in $x$ - and in $y$-direction is calculated.

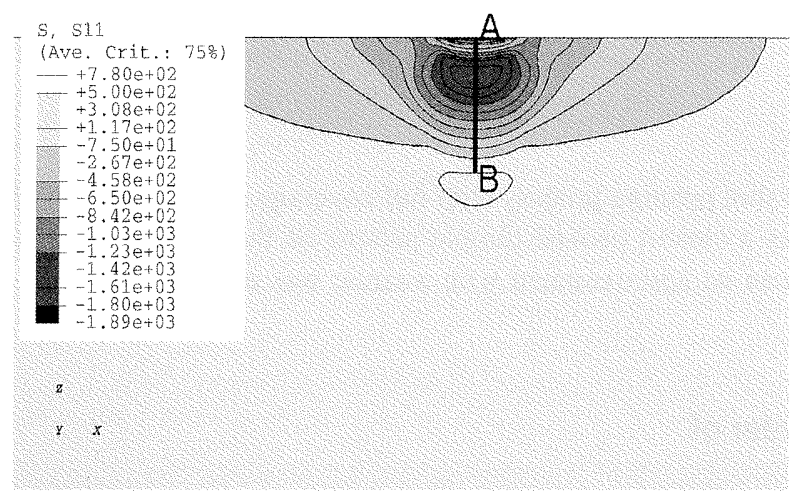

Figure 3: Residual stresses in $\mathrm{x}$-direction after 1 impact

\section{Results and Discussion}

\subsection{Effect of Adjacent Impacts}

In preliminary studies the effect of several successively impacting shots, set at a certain distance to each other, on the residual stress distribution was studied. Therefore, an impact of a single shot in the middle of the target model was simulated. The initial velocity of the shot was $35 \mathrm{~m} / \mathrm{s}$. This first impact was surrounded by 6 further impacts so that the borders of the remaining dimples had contact to their neighbors.

The residual stress distribution in $x$-direction after the first impact is represented in figure 3 . The maximum of residual stresses parallel to the surface occurs in a depth of approximately $0.05 \mathrm{~mm}$. In this region compressive residual stresses of $1600 \mathrm{MPa}$ are calculated. The remaining dimple diameter is $146 \mu \mathrm{m}$, its calculated depth is $9.4 \mu \mathrm{m}$.

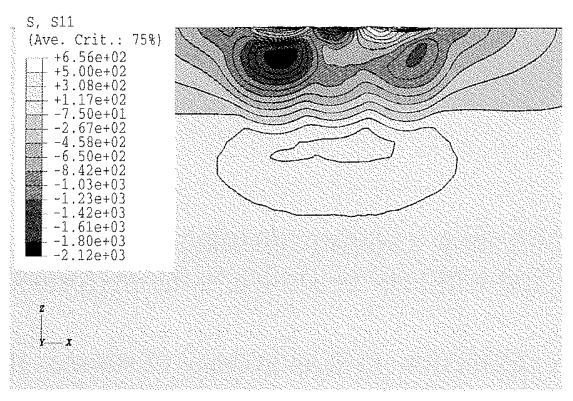

Figure 4: Residual stresses in $x$-direction after 7 impacts

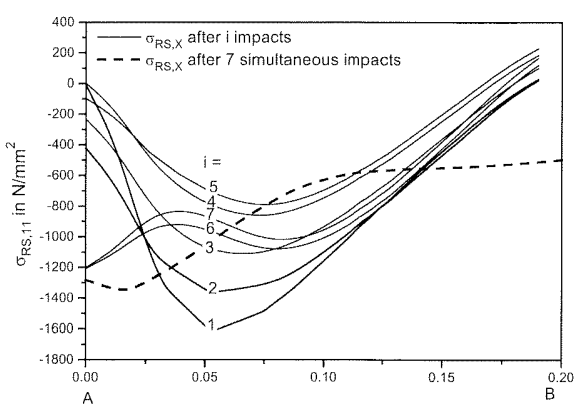

Figure 5: Residual stresses along the path $A B$ after the number of impacts given in the respective curve 
After the impact of the 6 further shots a redistribution of residual stresses parallel to the surface occurs (Fig. 4). The compressive residual stresses under the first impact get reduced by the impact of the following shots.

Figure 5 shows the development of residual stresses along the path $\mathrm{AB}$ from figure 3 after each impact. The first 4 succeding shots cause a remarkable reduction of the maximum compressive residual stress while the position of the stress maximum is shifted into the target material. The next impact causes an increase in compressive stresses with its maximum at $0.075 \mathrm{~mm}$ depth. It is also responsible for large compressive residual stresses at the surface. The last shot doesn't give a significant change of the stress state along the path.

These results show the interference of adjacent shot impacts. Succeding impacts have great influence on residual stresses under precedent impacts. In contrast to these results figure 6 presents the analysis of the 7 simultaneously impacting shots. In this case the stress state develops differently. The maximum of the compressive residual stresses is situated below the central dimple. Its value exceeds $-1300 \mathrm{MPa}$ and its position is closer to the surface. The respective residual stress profile is shown in figure 5 using a dashed line. At depths up to about $0.05 \mathrm{~mm}$ the workhardening induced during previous shots reduces the plastic strains and therefore the residual stresses induced. At higher depths the residual stresses after succeeding impacts are higher because the region of plastic strains was shifted to higher depths due to workhardening at small depths.

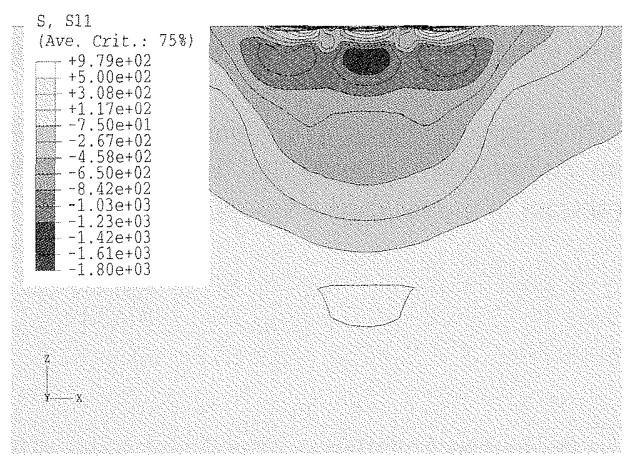

Figure 6: Residual stresses in $\mathrm{x}$-direction after 7 simultaneous impacts

\subsection{Analysis and Verification of Surface Layer Characteristics}

The above presented shot peening model was used to simulate the shot peening process. The shot-peening parameters were comparable to performed verification experiments conducted with an air-blast machine: A shot velocity of $35 \mathrm{~m} / \mathrm{s}$ and shot diameter of $0.56 \mathrm{~mm}$ was chosen. In the analysis all shots impacted perpendicular to the surface. Figure 7 presents the resulting residual stress profile compared to an experimentally obtained one measured at an AISI 4140 which was shot peened with similar parameters [8]. The calculated profile is of the same shape but it shows larger maximum compressive stresses $\sigma_{R S, \max }$ and larger surface stresses $\sigma_{R S, s u r f}$. The position of maximum compressive residual stresses $x_{\max }$ and of zero stresses $x_{0}$ is comparable to the experimental results. 


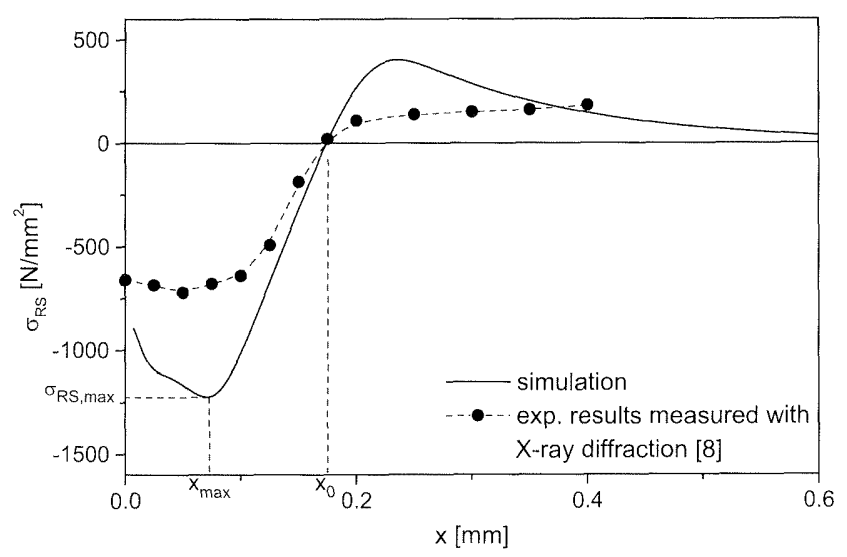

Figure 7: Calculated and experimental residual stress pofile

\subsection{Effect of Coverage}

In order to determine if the chosen dimple configuration can be compared to 100 percent coverage further 19 spheres were added to the model. Their impact location has been changed by rotating the inner and outer circles so that the center of impact of the last 19 spheres was placed on the point of contact of two former dimples.

The resulting residual stress profiles doesn't show significant change compared to the 19shot-model. Only a slight increase in maximum compressive stresses can be noticed. This shows that the 19-shot-model achieves a saturation state of the residual stress profile which isn't significantly changed by further impacts.

\subsection{Effect of Shot Velocity}

The effect of shot velocity on residual stresses was studied by calculation of residual stress profiles resulting from different shot velocities (Fig. 8). With increasing shot velocity there is no significant change in calculated surface and maximum compressive stresses. The position of maximum compressive stresses moves into the material with increasing shot velocity. The same behavior is shown by the zero-crossing of the stress profile.

These results are in good agreement with experimental results obtained by variing the pressure in an air blasting machine [9].

\subsection{Effect of Shot Diameter}

The effect of variing the shot diameter on residual stress profiles was calculated using a constant shot velocity of $35 \mathrm{~m} / \mathrm{s}$. The analysis results show a strong dependence of the position of the maximum compressive stress on shot diameter (Fig. 9). The zero-crossing of the stress profiles does also move into the target with increasing shot diameter. In contrast to that the surface 


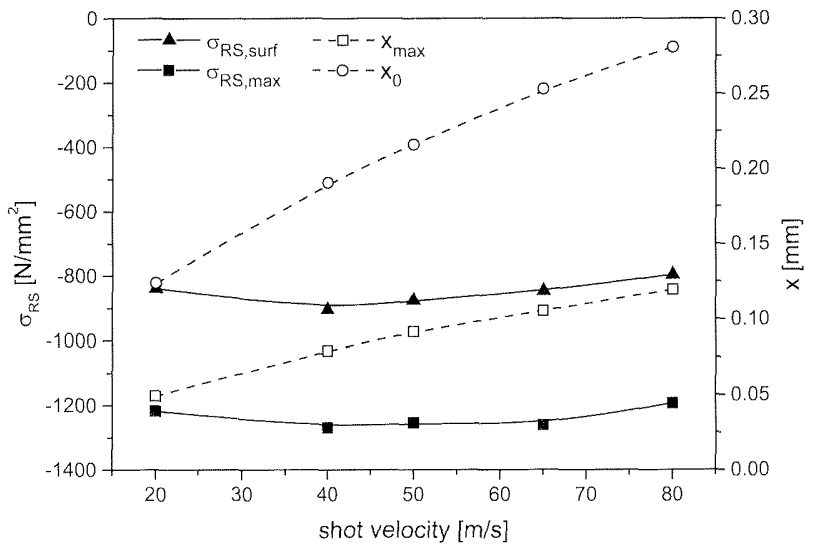

Figure 8: Influence of shot velocity on the residual stress profile

residual stresses and the maximum compressive residual stresses don't show any dependence on shot diameter. Experimental results from [9] also show an increase of $x_{0}$ with growing shot diameter whereas $\sigma_{R S, \max }$ is not affected from the shot size.

\subsection{Effect of Impact Angle}

To study the influence of the impact angle on the residual stress profile the impact angle was variied at a constant shot velocity of $35 \mathrm{~m} / \mathrm{s}$. Within an analysis each of the 19 spheres had the same direction. The calculated results show a decrease of surface and maximum residual compressive stresses with increasing impact angle. The position of maximum compressive residual

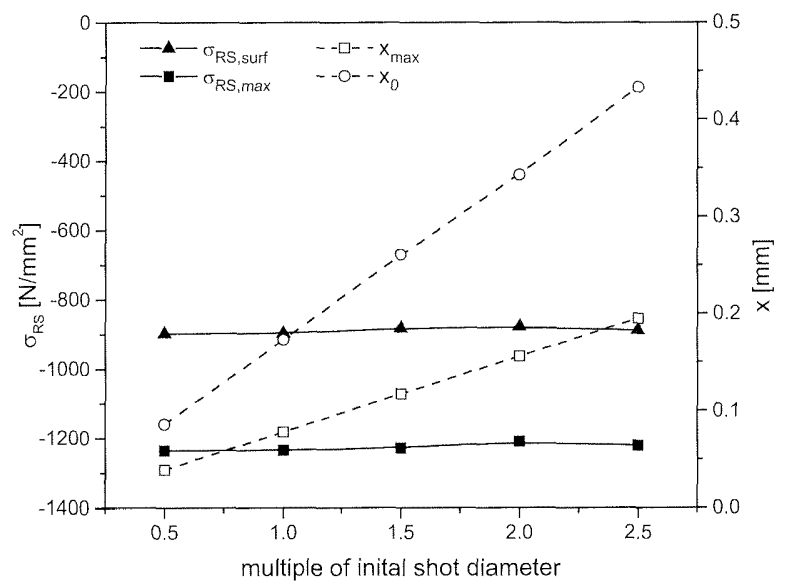

Figure 9: Influence of shot diameter on the residual stress profile 


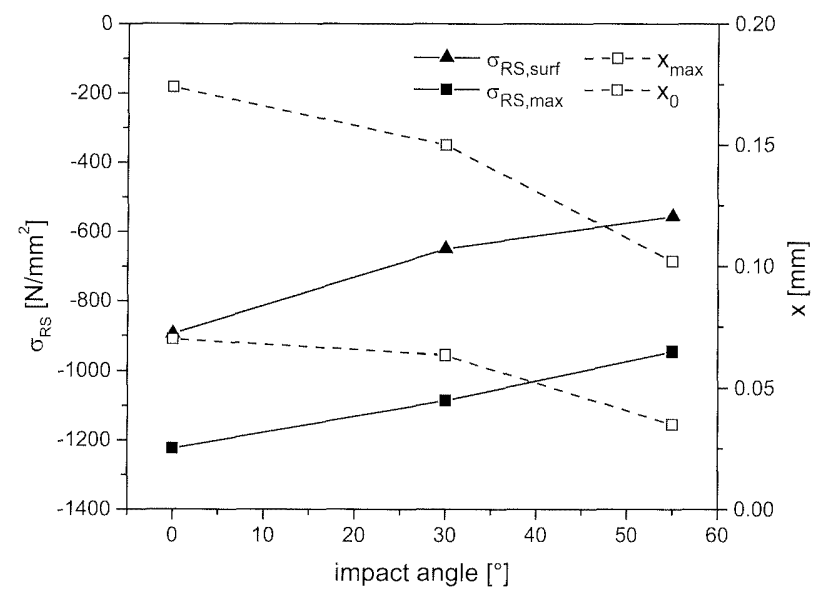

Figure 10: Influence of the impact angle on residual stress profiles

stresses and the zero-crossing of the residual stress profile also show a strong dependence on the impact angle. Experiments conducted with an air-blast machine variing the peening angle of a quenched plain carbon steel show the same effect [10].

\section{Conclusions}

A three-dimensional finite element model was developed to simulate the shot peening process. Multiple dynamic spherical indentations were examined and a method for the calculation of residual stress profiles was presented. The effect of shot velocity, diameter, coverage and impact angle upon residual stress profiles was examined and discussed. The results reveal that the depth of the compressed layer is significantly increased by growing shot velocity or shot diameter. A significant influence on surface and maximum compressive residual stresses could not be shown. An increase in the impact angle reduces surface and maximum residual stresses as well as the depth of the residual stresses.

The current work indicates that the proposed finite element model is capable to capture the shot peening process, thus implying its potential as an effective tool for the prediction of residual stress profiles.

\section{$5 \quad$ References}

[1] S. A. Meguid, G. Shagal, J. C. Stranart, Finite Elements in Analysis and Design, 1999, 31, 179-191.

[2] K. Schiffner, C. Droste gen. Helling, Computers and Structures, 1999, 72, 329-340.

[3] K. Han, D. Periæ, A. J. L. Crook, D. R. J. Owen, Engineering Computations, 2000, Vol.17, No.5, 593-619. 
[4] S. T. S. Al-Hassani, K. Kormi, K. Webb, D. C. Webb, Proceedings ICSP 7, 1999, 217-226.

[5] V. Schulze, O. Vöhringer, Metallurgical and Materials Transactions A, 2000, 31A, 825830.

[6] C. Tome, G. R. Canova, U. F. Kocks, N. Christodoulou, J. J. Jonas, Acta metall. 1984, Vol. 32, No.10, 1637-1653.

[7] B. Scholtes, Eigenspannungen in mechanisch randschichtverformten Werkstoffzuständen, Ursachen-Ermittlung-Bewertung, DGM-Informationsgesellschaft, Oberursel, 1990.

[8] R. Menig, Dissertation, University of Karlsruhe, in preparation.

[9] A. Wick, H. Holzapfel, V. Schulze, O. Vöhringer, Proceedings ICSP 7, 1999, 42-53.

[10] A. Ebenau, O. Vöhringer, E. Macherauch, Proceedings ICSP 3, 1987, 253-260. 